

\title{
Development of a CCD-based fluorimeter for real-time PCR machine
}

\author{
Da-Sheng Lee ${ }^{\mathrm{a}, *}$, Bou-How Chang ${ }^{\mathrm{b}}$, Ping-Hei Chen ${ }^{\mathrm{b}}$ \\ ${ }^{a}$ Department of Air Conditioning and Refrigeration, National Taipei University of Technology, Taipei 106, Taiwan \\ ${ }^{\mathrm{b}}$ Department of Mechanical Engineering, National Taiwan University, Taipei 106, Taiwan
}

Received 29 April 2004; received in revised form 10 December 2004; accepted 10 December 2004

Available online 19 January 2005

\begin{abstract}
A monochromic charged coupled device (CCD) is employed to obtain time evolved fluorescence images that are reflected from PCR mix in a $20 \mu \mathrm{L}$ capillary during thermal cycling in the real-time polymerase chain reaction (real-time PCR). This fluorescence detection system consists of a $470 \mathrm{~nm}$ light emitting diode (LED) as an excitation light source, a CCD camera, and a rotating disk with six optical filters to provide six fluorescence detection channels at 530, 560, 610, 640, 670 and $710 \mathrm{~nm}$ for multiplex real-time PCR DNA quantification. In this study, the PCR mix that contains HBV SC 11 DNA template with SYBR Green I fluorescence labeling dye is tested to verify the prototype performance. An adaptive image processing scheme based on a lower-upper-middle (LUM) filter algorithm is developed to eliminate noise while keeping the necessary information from recorded fluorescence images. The measured results obtained by the proposed image processing scheme illustrate that the real-time PCR prototype with CCD-based fluorimeter can achieve similar DNA quantification reproducibility as the commercial machine even when the initial DNA copies, $X$, in the tested PCR mix is down to $10^{3}$ copies $/ \mathrm{mL}(0.01 \mathrm{fM})$.
\end{abstract}

(C) 2004 Elsevier B.V. All rights reserved.

Keywords: Real-time PCR; CCD; Fluorescence detection system; LUM filter; Fluorescence image processing

\section{Introduction}

The PCR [1-3] process was first introduced in 1986 by Mullis and others to replicate the specified DNA fragments in vitro. Since then, the PCR process has become one of the major tools in genomic studies. There are three major steps in a single thermal cycle of PCR that usually requires 20-30 thermal cycles. The first step is called "denaturation" during which the PCR mix in a tube is heated up to $95^{\circ} \mathrm{C}$ and the double-strand DNA breaks. The second step is called "annealing" that occurs as the temperature of PCR mix drops down to about $53^{\circ} \mathrm{C}$. Primers with an approximate length of $15-30 \mathrm{bp}$ are jiggling around in the mix by Brownian motion and are attached to single-strand DNA with ionic bonds. The

\footnotetext{
* Corresponding author. Tel.: +886 928868885; fax: +886 227988272 .

E-mail address: f11167@ntut.edu.tw (D.-S. Lee).
}

final step is called "elongation" that happens at an approximate temperature of $72{ }^{\circ} \mathrm{C}$. It is the ideal working temperature for the polymerase. Since the primers already have strong ionic bonding to the DNA template at specific position, the polymerase will add dNTP's from $5^{\prime}$ to $3^{\prime}$ to the template by reading the template from $3^{\prime}$ to $5^{\prime}$ end. The specific DNA sequence is amplified exponentially with thermal cycling.

By integrating a fluorimeter with the thermal cycler, a realtime PCR machine was first introduced in 1997 [4,5]. This real-time PCR machine allows the detection of DNA amplification through the detection of the fluorescence labeling dye in the PCR mix during the early phase of the reaction. In addition, the concentration of target DNA fragment in the PCR mix before thermal cycling can be obtained from the time recorded history of the fluorescence intensity. The realtime PCR machine has high sensitivity and consumes less time when compared to the traditional PCR machine. In addition, there is no need to perform agarose gel electrophore- 
sis on PCR mix after thermal cycling from the real-time PCR machine to ensure successful amplification on DNA template that is inevitable for amplified PCR mix from the traditional PCR machine.

The current commercial real-time PCR machine has three discrete channels with photodiodes for detecting fluorescence emitted from the PCR mix. In order to separate different fluorescence wavelengths, one set of narrow band filters and dichromatic mirrors are used to allow fluorescence with the required wavelength to reach the corresponding channel. Since the emitting dye fluorescence has to pass through complicated optical path before it reaches the appropriate channel, the intensity is decayed to $40-50 \%$ of the fluorescence intensity detected by the previous channel. Therefore, it is impossible to have the capability to detect five different dyes in one sample of the PCR mix due to significant optical loss.

However, simultaneous detection of multiple target DNA sequences becomes a trend in either virus or blood inspection. The detection of different viruses in one blood sample is called multiplex real-time PCR. It has become the aim for many researchers. Some approaches were proposed to achieve multiplex PCR by using a biochip solution [6,7]. Some employed the multi-channels fluorescence detection $[8,9]$ to achieve the capability for multiplexing PCR. Wittwer et al. [8] employed a spectrograph with continuous spectral dispersion and a line CCD array detector to monitor the fluorescence time history during thermal cycling of a real-time PCR machine. The spectrograph with a concave holographic grating can normally achieve a $25-35 \%$ collection efficiency in $300-800 \mathrm{~nm}$ fluorescence wavelength. The line CCD array detector has to be cooled to $-70^{\circ} \mathrm{C}$ for achieving high sensitivity on spectral distribution of the fluorescence. Thus, the whole system with the spectrograph is too big and expensive. There is one commercial machine [9] with a photo demultiplexer that can separate six optical wavelengths from one fluorescence input towards the corresponding photodiode detector. Through this sequential optical separation, the fluorescence collection efficiency can exceed $70 \%$ for each optical channel. With such a high signal level, the photodiode can be used as the optical detector. Although this solution can provide a multi-channel detection capability, the reliability of this kind of real-time PCR machine is still under evaluation as the photo demultiplexer is not a mature product yet [10-14]. In addition, the cost of demultiplexer is expensive.

Our previous study was to build up a real-time PCR machine with a miniature spectrometer for spectral dispersion of emissive fluorescence [15]. Test results show that the system provides comparable accuracy and reproducibility as the current commercial machine for DNA quantification and the system is compact comparing with the one suggested by Wittwer et al. However, this kind of solution is still expensive for laboratory usage.

In order to obtain a compact and inexpensive system for prevalent application, this study aims to develop a real-time
PCR prototype with an inexpensive fluorescence detection system. A CCD detector can offer a wide range of spectral response that can be divided by a sliding interference optical filter into fluorescence detection channels at 530, 560, 610, 640, 670 and $710 \mathrm{~nm}$ wavelengths by six interference filters on the rotating holder. This kind of optical engine design is simple and does not need the precision housing structure like the one employed by commercial real-time PCR machine or the miniature spectrometer used in our previous study. It is an inexpensive and convenient solution provided that the performance of the prototype should be comparable with the commercial real-time PCR machine.

Since the design of this prototype fluorescence detection system is different from our previous study, an analytical model based on Beer-Lambert law [16-19] is developed to predict the fluorescence intensity that increases with the amplification of DNA concentration during PCR thermal cycling in this prototype. The predicted values can be used as a basis for determining DNA initial copies in PCR mix and identifying the noise characters of the results that are obtained through a signal filtering algorithm over fluorescence images captured by the CCD detector. In this study, an adaptive filter with LUM algorithm is employed to eliminate optical noise in the captured fluorescence images due to the high noise level of the CCD detector.

The key performance indices of real-time PCR machine are DNA quantification sensitivity and reproducibility $[20,21]$. The DNA quantification sensitivity depends not only on the machine itself but also on the selection of PCR primer and fluorescence labeling dye. In this study, the machine performance is evaluated by using known initial DNA template concentration in the PCR samples for DNA quantitative experiments in both the commercial real-time PCR machine and the prototype. Single DNA template, HBV SC 11, with known concentrations and a single labeling dye, SYBR green I, are used in PCR mix. A comparison will be presented to show that similar sensitivity in DNA quantification is achieved in both machines.

By repetitive determinations of known concentrations of DNA template in the same run or in different runs in the realtime PCR machine, the coefficient of variation (CV) of intraassay for the same run or inter-assay for different runs can be determined, respectively. Since the uncertainty in human operation will affect the CV value of inter-assay measurements, the $\mathrm{CV}$ value of intra-assay runs is employed in this study to evaluate the real-time PCR prototype performance. A lower $\mathrm{CV}$ value indicates higher reproducibility. The $\mathrm{CV}$ values of intra-assay at different initial DNA copies are obtained for both the commercial real-time PCR machine and the prototype.

\section{Experimental apparatus}

Two real-time PCR machines, the prototype and a commercial machine (Roche, Light Cycler, USA), were tested in 
this study for quantitative measurements on the concentration of HBV SC 11, DNA fragment. The initial DNA copy in PCR mix ranged from $10^{3}$ to $10^{9}$ copies $/ \mathrm{mL}$. LightCyclerFast Start DNA master with Cat No. 3003230 and SYBR Green I labeling dye were employed as the PCR mix in all runs. The test sample was loaded into a $20 \mu \mathrm{l}$ capillary tube and sealed with a plastic plug. The inner diameter of the capillary tube was $0.8 \mathrm{~mm}$ and the outer diameter was $1.15 \mathrm{~mm}$. The prototype used in this study is similar to that in the paper of Lee et al. [15] but the spectrometer in their prototype was replaced with a CCD camera and a sliding filter in this study. Fig. 1 shows details of the thermal cycling chamber. The thermal cycling condition is stated as follows: It requires a $10 \mathrm{~min}$ incubation at $95^{\circ} \mathrm{C}$. After the incuba- tion, each thermal cycle undergoes $3 \mathrm{~s}$ of $95^{\circ} \mathrm{C}$ denaturation, $10 \mathrm{~s}$ of $53{ }^{\circ} \mathrm{C}$ annealing, and $16 \mathrm{~s}$ of $72^{\circ} \mathrm{C}$ elongation to amplify the DNA fragment. The chamber temperature can be maintained precisely with a variation of $\pm 0.6^{\circ} \mathrm{C}$ at $94^{\circ} \mathrm{C}$, a variation of $\pm 0.4^{\circ} \mathrm{C}$ at $56^{\circ} \mathrm{C}$, and a variation of $\pm 0.6^{\circ} \mathrm{C}$ at $72^{\circ} \mathrm{C}$ without any significant overshooting or undershooting of the chamber temperature. Since Lee et al. [15] provided a detailed description of the thermal cycling system for both the commercial real-time PCR machine and the prototype, here we only describe the design of our optical detector.

A monochromic CCD (Watec Inc., WAT-902H, JPN) was used to measure a wide spectral response for fluorescence intensity reflected from the PCR mix. The prototype has a

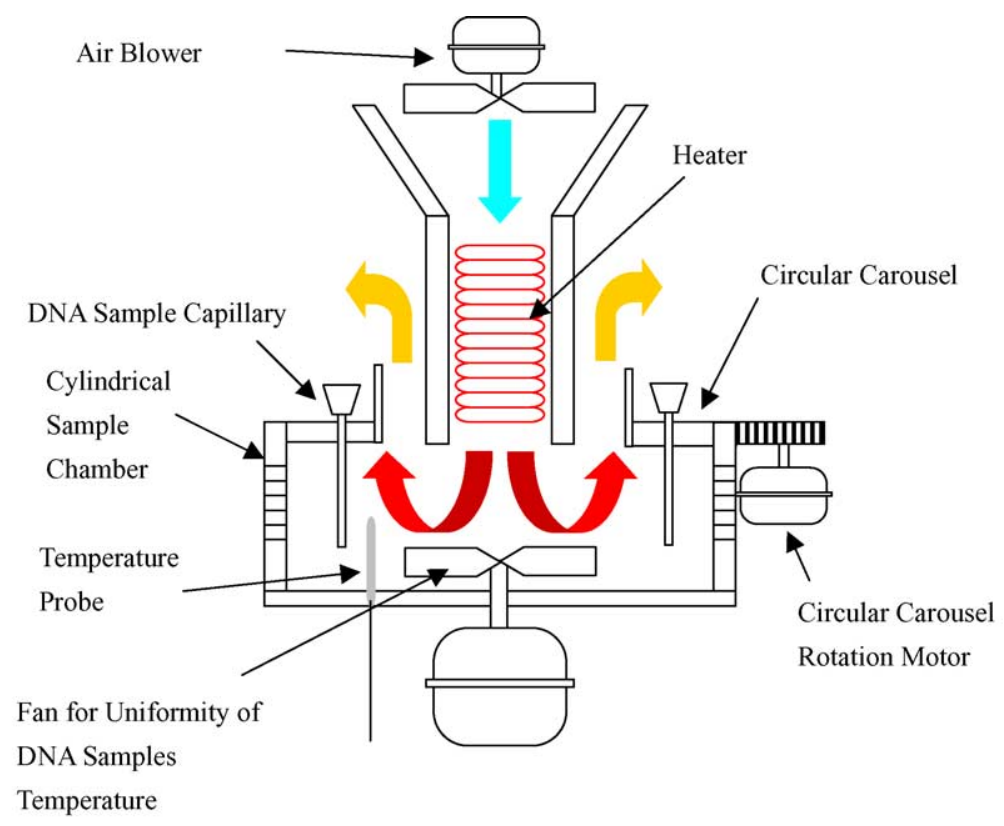

(a)

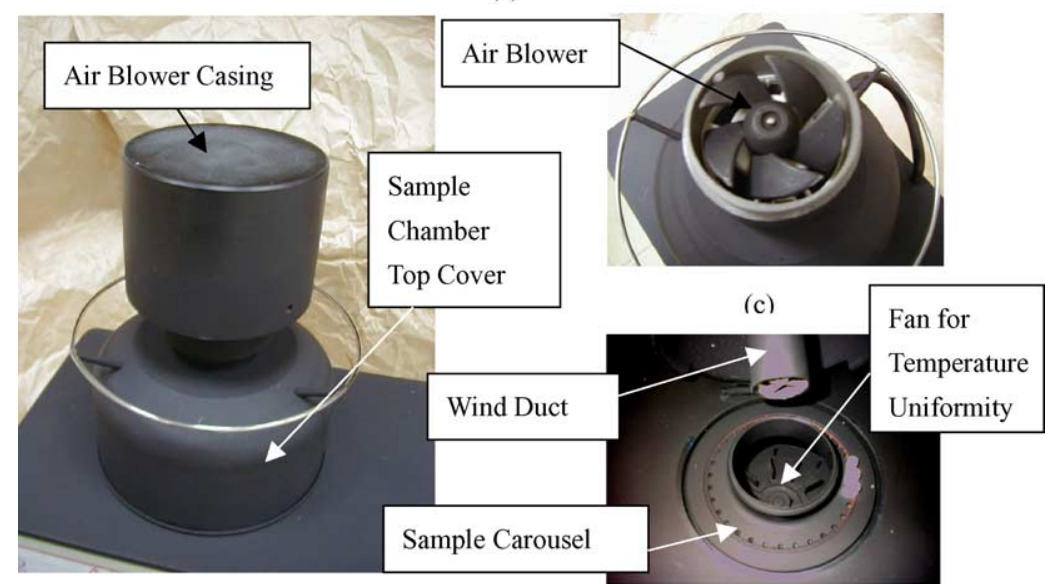

(b)

(d)

Fig. 1. The thermal cycling system of the present prototype (a) schematic view, (b) the photograph of the system, (c) the air blower, (d) the arrangement of the wind duct with heater inside, DNA samples carousel and the fan for achieving uniformity in DNA samples temperature. 


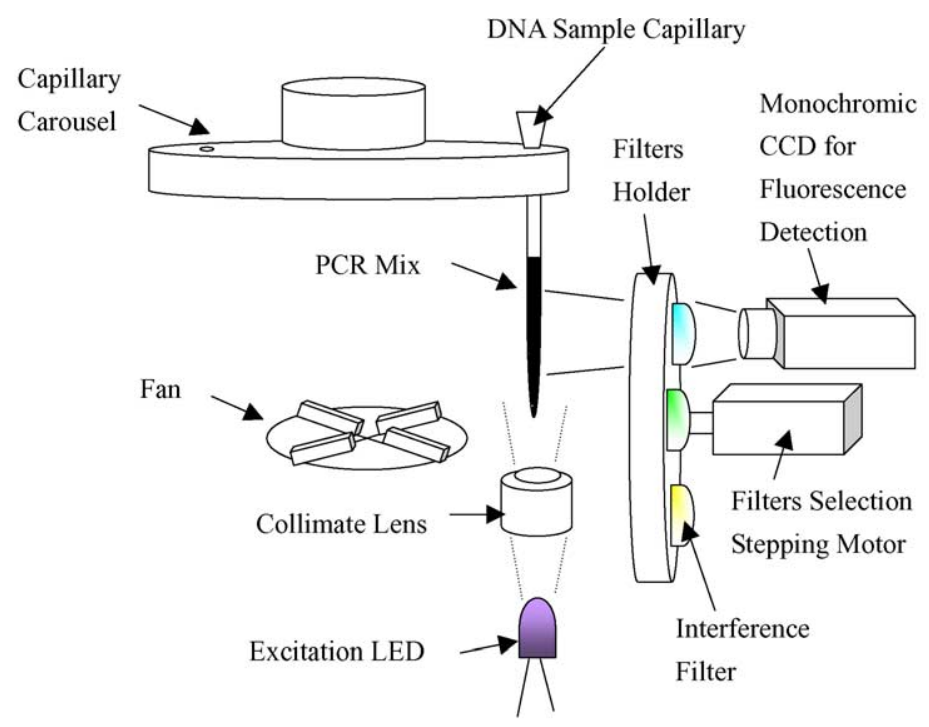

(a)



Fig. 2. The fluorescence detection system (a) the schematic view, (b) the optical engine photograph, (c) the arrangement of the DNA sample capillary with PCR mix, the excitation light source and the sliding filters.

capability of six detection channels at 530, 560, 610, 640, 670 and $710 \mathrm{~nm}$ by using six $1.0 \mathrm{in}$. diameter interference filters (UNICE E-O Services Inc., Part No. CWL-F10-25.4, TWN) with $10 \mathrm{~nm}$ cut off limit around the central wavelength. A LED (UNICE E-O Services Inc., Part No. RL5-B2430, TWN) was employed as the excitation light source with peak emission at a wavelength of $470 \mathrm{~nm}$ and luminous intensity of $2400 \mathrm{mcd}$. The fluorescence detection system is shown in Fig. 2.

\section{Model simulation and determination of initial DNA copies in PCR mix}

Since the design of optical sensing device for fluorescence is different from the commercial real-time PCR machine and our previous study, a new simulation model is introduced to predict the reflected fluorescence from PCR mix with PCR thermal cycling. Predicted fluorescence signals are obtained and they are functions of initial DNA concentration in the PCR mix and thermal cycling number of PCR. The model simulation results are used to determine the initial copies of target DNA before thermal cycling from the time history of fluorescence intensity that increases with real-time PCR thermal cycle.

\subsection{Predicted emissive fluorescence intensity for the real-time PCR prototype}

A general expression for emissive fluorescence from a PCR mix based on Beer-Lambert law can be given by

$$
\begin{aligned}
\Delta F= & I_{\lambda}(0) \int_{0}^{L} \int_{0}^{2 \pi} \int_{\varphi_{1}(x, \theta, \varphi)}^{\varphi_{2}(x, \theta, \varphi)} \sin \varphi \sum_{i=1}^{N} y_{i} a_{i, \lambda} c_{i}\left(\frac{I_{\lambda}(x)}{I_{\lambda}(0)}\right) \\
& \times\left(\frac{I_{\lambda^{\prime}}(x)}{I_{\lambda^{\prime}}(0)}\right) \mathrm{d} \varphi \mathrm{d} \theta \mathrm{d} x
\end{aligned}
$$

where $\Delta F$ denotes fluorescence signal intensity that is proportional to multiplication of $I_{\lambda}(0)$, excitation light intensity, and labeling dye quantum yield $y_{i} a_{i, \lambda}$ denotes molar extinction coefficient and $c_{i}$ denotes DNA template concentration at $i$ th PCR thermal cycle. Two fractional terms $\left(I_{\lambda}(x) / I_{\lambda}(0)\right)$ and $\left(I_{\lambda^{\prime}}(L) / I_{\lambda^{\prime}}(0)\right)$ denote excitation light decay due to the absorption of labeling dye and emissive fluorescence decay 




Fig. 3. Definitions of the core angle, the solid angle and the integration length for the real-time PCR prototype with CCD-based fluorescence detection system.

as excitation light and emissive fluorescence pass through the DNA sample in a capillary tube with a sample length of $L$. Definitions of core angle, solid angle and integral length of the real-time PCR prototype with a CCD-based fluorescence detection system can be referred to in Fig. 3. The integration is performed along the DNA sample length, around the whole core angle $\theta$ from 0 to $2 \pi$, and over the solid angle $\varphi$ from $\varphi_{1}$ to $\varphi_{2}$. Note that both upper and lower limits of solid angle vary with the design of optical sensing device. The summation index, $N$, depends on the number of different DNA labeling dyes in the PCR reagent. The model was verified by a comparison between measured and predicted results of the commercial real-time PCR machine with different initial copies of HBV SC11 DNA in the PCR mix. The details can be referred to in our previous study [15].Due to the different arrangements in optical sensing device of the prototype and the commercial one, the emissive fluorescence decay should be revised as:

$\left(\frac{I_{\lambda^{\prime}}(L)}{I_{\lambda^{\prime}}(0)}\right)=1-0.75\left(-\mathrm{e}^{-L a_{i, \lambda} c_{i}}+1\right)$

because fluorescence light directly transports through the capillary tube wall and about one fourth of the light can be collected by the detector.

Substituting Eq. (2) into Eq. (1),

$$
\begin{aligned}
\Delta F= & 2 \pi I_{\lambda}(0) \int_{0}^{L} \int_{0}^{\pi / 12} \sin \varphi y_{i} a_{i, \lambda} c_{i} \mathrm{e}^{-x a_{i, \lambda} c_{i}} \\
& \times\left(1-0.75\left(-\mathrm{e}^{-L a_{i, \lambda} c_{i}}+1\right) \mathrm{d} \varphi \mathrm{d} x\right.
\end{aligned}
$$

The core angle $\varphi^{\prime}$ is confined by the distance between the DNA sample tube and the CCD collection lens, as shown in Fig. 3. The value of solid angle can be obtained as $\pi / 12$. The integral can then be derived as:

$$
\begin{aligned}
\frac{\Delta F}{I_{\lambda}(0)}= & 2 \pi \cdot 0.0102222\left(1-\mathrm{e}^{-L a_{i, x} c_{i}}\right) \\
& \times\left(1-0.75\left(-\mathrm{e}^{-L a_{i, x} c_{i}}+1\right)\right)
\end{aligned}
$$

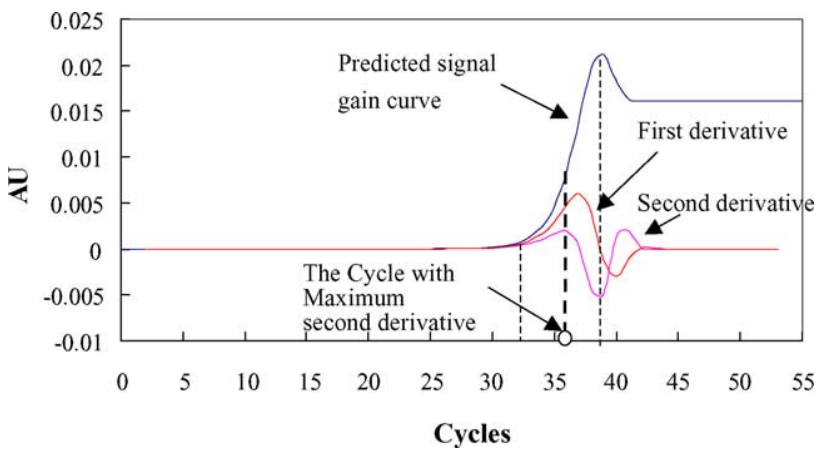

Fig. 4. Predicted signal gain $\left(\Delta F / I_{\lambda}(0)\right)$, its first and second derivatives with initial $10^{5}$ copies of DNA in $1 \mathrm{~mL}$ for the RT-PCR prototype.

\subsection{Determination of initial DNA copies in PCR mix for the real-time PCR prototype}

Some important biomolecular information can be obtained from signal processing on fluorescence intensity reflected from the PCR mix. Such information includes the specificity of target DNA, the initial copies of target DNA, etc. The aim of this study is to determine the initial copies of target DNA before thermal cycling from the time history of fluorescence intensity that increases with real-time PCR thermal cycle. If the PCR efficiency is assumed perfect at each cycle, the concentration of target DNA in the mix at $i$ th PCR cycle can be expressed as the following:

$c_{i}=\frac{N}{6.02 \times 10^{20}} 2^{i}$

where $N$ denotes the initial DNA template copies/ml. When divided by the constant, it can be expressed by the concentration unit.

The predicted fluorescence intensity curve for the RT-PCR prototype and its first and second derivatives versus thermal cycle are shown in Fig. 4. There are two local maximum values in the second derivative of predicted fluorescence intensity because the fluorescence intensity drops with thermal cycle number after it reaches saturation. To avoid nonspecific amplification of DNA caused by excessive PCR thermal cycles, the thermal cycle number corresponding to the first maximum value in the second derivative should be considered as the critical cycle number for determination of the initial DNA copies. Similar approach can be derived for the prototype to find a linear relationship between the reciprocity of critical cycle number and the log value of initial DNA copies.

Replacing $a_{i, \lambda} c_{i}$ by $C_{i}$ in Eq. (3), it yields

$$
\Delta F=\operatorname{const}\left(1+2 \mathrm{e}^{-L C_{i}}-3 \mathrm{e}^{-2 L C_{i}}\right)
$$

The second derivative of the fluorescence intensity reaches a local maximum value when the third derivative of the fluorescence intensity equals zero. The third derivative can be 
expressed as:

$$
\begin{aligned}
\frac{\mathrm{d}^{3} F}{\mathrm{~d} i^{3}}= & \text { const }\left[\left(2 L^{2} \mathrm{e}^{-L C_{i}}-4 L^{2} \mathrm{e}^{-2 L C_{i}}\right) \frac{\mathrm{d} C_{i}}{\mathrm{~d} i}\right. \\
& \left.+\left(-2 L \mathrm{e}^{-L C_{i}}+2 L \mathrm{e}^{-2 L C_{i}}\right) \frac{\mathrm{d}^{2} C_{i}}{\mathrm{~d} i^{2}}\right]
\end{aligned}
$$

It equals zero only if

$$
\begin{aligned}
& \left(2 L^{2} \mathrm{e}^{-L C_{i}}-4 L^{2} \mathrm{e}^{-2 L C_{i}}\right)+\left(-2 L \mathrm{e}^{-L C_{i}}+2 L \mathrm{e}^{-2 L C_{i}}\right) \\
& \ln 2=0
\end{aligned}
$$

The solution of Eq. (8) is given by

$\mathrm{e}^{L C_{i}}=\frac{4 L^{2}-2 L \ln 2}{2 L^{2}-2 L \ln 2}$

By taking $\log$ on both sides of Eq. (9) and substituting $C_{i} \propto$ $N 2^{i}$ into Eq. (9), it yields

$$
C_{i_{\mathrm{c}}}=N 2^{i_{\mathrm{c}}}=\frac{1}{L} \ln \left(\frac{4 L^{2}-2 L \ln 2}{2 L^{2}-2 L \ln 2}\right)=\mathrm{const}
$$

where $i_{\mathrm{c}}$ denotes the critical cycle number at which the second derivative of fluorescence intensity first reaches its local maximum value. Note that the second derivative of fluorescence intensity reaches a local maximum value at two different thermal cycle number for the prototype.

Furthermore,

$N \propto$ const $\frac{1}{2^{i_{\mathrm{c}}}}=$ const $\frac{1}{\mathrm{e}^{i_{\mathrm{c}} \ln 2}}$

and

$\log N \propto C_{\mathrm{c}} \frac{1}{i_{\mathrm{c}}}$

It indicates that a linear relationship exists between the reciprocity of critical cycle number and the log value of initial DNA copies. Based on the equation, the coefficient of linear equation, $C_{\mathrm{c}}$, can be determined from a set of PCR samples with known copies of target DNA. Once the coefficient is obtained, the unknown copies of target DNA in tested samples can be determined from Eq. (12) for the prototype.

\section{Signal filtering scheme for fluorescence image captured by the CCD detector of the prototype}

From the predicted results by the optical model for the prototype described above, the real-time PCR prototype with CCD-based fluorimeter can be employed for determination of initial DNA copies by recording the time history of fluorescence images of each cycle of PCR. However, poor image quality with significant noise may reduce accuracy in determining initial copies of tested DNA. Therefore, an adaptive signal filtering scheme based on a LUM algorithm is introduced to eliminate signal noise.

\subsection{LUM filter}

A filter algorithm, called lower-upper-middle (LUM) filter algorithm, is based on the comparison between lower order statistics, middle order statistics and upper order statistics to determine the signal intensity output. If optimal parameters can be chosen, the LUM algorithm is good at eliminating noise while keeping the useful information of the fluorescence image.

Considering the fluorescence image over the measured area with $M$ pixels, it requires a matrix of for processing the grayscale of original image. The total number of elements, $k$ or $l$, in the matrix must be chosen as an odd number. The matrix can be expressed as:

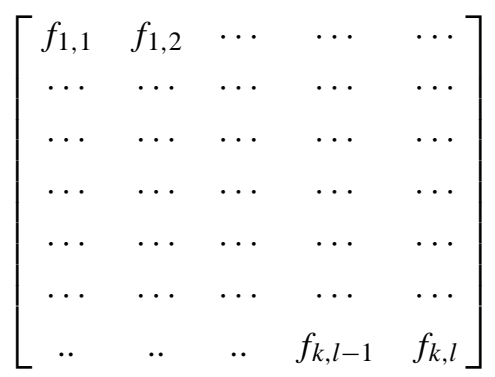

where $f_{*}, *$ is the grayscale at a specified pixel location that required to be converted. The grayscale of the image over the measured area can be expressed in sequence, and is given by

$W=\left\{f_{(1)}, f_{(2)}, \ldots, f_{(M-1)}, f_{(M)}\right\}$

where $f_{(1)}<f_{(2)}<\cdots<f_{(M-1)}<f_{(M)}$. Before the converting process on grayscale of captured image, two parameters, $o$ and $p$, must be defined for determining the lower order statistics and upper order statistics. In this study, $o<p<(M+1) / 2$. The lower and upper order statistics are defined as

$$
\begin{aligned}
& S_{L}=\operatorname{med}\left\{f_{(o)}, f_{*}, f_{(p)}\right\} \\
& S_{U}=\operatorname{med}\left\{f_{(M-o+1)}, f_{*}, f_{(M-p+1)}\right\}
\end{aligned}
$$

The med calculation algorithm is defined as

$$
\begin{array}{ll}
\text { If } & f_{*}<f_{(o)} \text { then } S_{L}=f_{(o)} \\
\text { Else } & \text { If } \\
f_{(M-o+1)}<f_{*} \text { then } S_{L}=f_{(M-o+1)} \\
\text { Else } \quad S_{L}=f_{*}
\end{array}
$$

The upper statistics can be determined by the same med expression as that for obtaining lower statistics.

After both lower and upper order statistics are obtained, the middle order statistics can be found by

$S_{M}=\frac{1}{2}\left(S_{L}+S_{U}\right)$ 
The algorithm for determining the grayscale at specified pixel location of processed image is

$$
g_{*}= \begin{cases}f_{(o)} & \text { if } f_{*}<f_{(o)} \\ f_{(p)} & \text { if } f_{(o)}<f_{*} \leq S_{M} \\ f_{(M-p+1)} & \text { if } S_{M}<f_{*}<f_{(M-p+1)} \\ f_{(M-o+1)} & \text { if } f_{(M-o+1)}<f_{*} \\ f^{*} & \text { others }\end{cases}
$$

\subsection{Adaptive signal processing scheme}

The LUM filter algorithm introduces too many pixels into calculations where no fluorescence signal appears. An adaptive signal pre-processing scheme is proposed to choose significant pixels to form the matrix before conversion on grayscale from the original image into the processed image. The image recognition scheme is based on the cross correlation of pixel grayscales between two horizontal neighboring lines. With respect to pixel grayscale on the first horizontal line, the covariance can be calculated by

$\mu_{j}=\frac{1}{W} \sum_{i=1}^{W} f_{i, j}, \quad \sigma_{j}^{2}=\frac{1}{W} \sum_{i=1}^{W}\left(f_{i, j}-\mu_{j}\right)^{2}$

The covariance of pixel grayscale on the second horizontal line is calculated by

$\mu_{j+1}=\frac{1}{W} \sum_{i=1}^{W} f_{i, j+1}$,

$\sigma_{j+1}^{2}=\frac{1}{W} \sum_{i=1}^{W}\left(f_{i, j+1}-\mu_{j+1}\right)^{2}$

The cross-correlation coefficient of pixel grayscale on two neighboring $\mathrm{p}$ lines can be determined by

$\hat{\sigma}_{j, j+1}=\frac{(1 / W) \sum_{i=1}^{W}\left(f_{i, j}-\mu_{j}\right)\left(f_{i, j+1}-\mu_{j+1}\right)}{\sigma_{j} \sigma_{j+1}}$

If the cross-correlation coefficient is larger than a critical value, the grayscale values on the second horizontal line will be included into the matrix for the LUM filter algorithm.

\section{Results and discussions}

The target DNA fragment, HBV SC 11, was employed for the DNA quantification measurements. The number of initial copies of target DNA ranged from $10^{3}$ to $10^{9} / \mathrm{mL}$. Samples were prepared using the LightCycler-Fast Start DNA master with Cat No. 3003230 and SYBR Green I labeling dye in all runs. The quantification experiments were performed on both the commercial real-time PCR machine and the prototype. The measured results were compared with the predicted fluorescence intensity by the proposed models. For the prototype, the fluorescence signal quality obtained from the

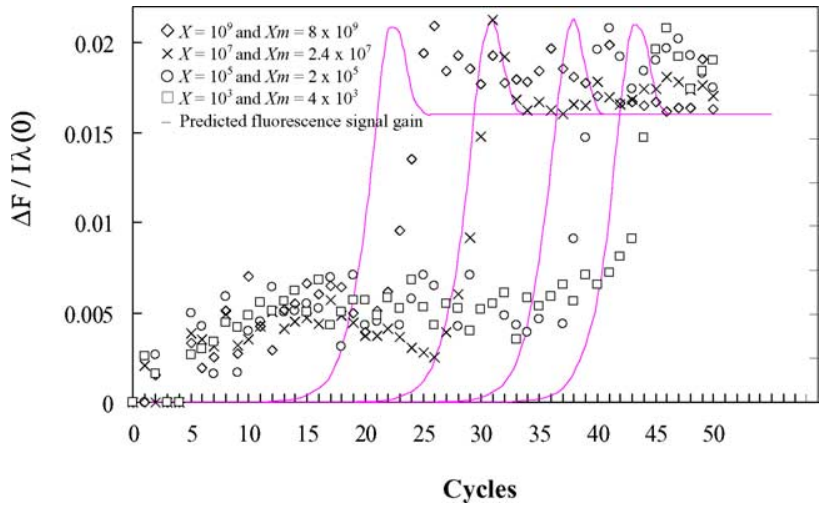

Fig. 5. The comparison of the measured fluorescence intensity calculated from the row fluorescence image with four different initial DNA copies denoted in each curve and fluorescence modeling predicted values.

CCD detector was not good enough for DNA quantification when the measured data were compared with the predicted value from the proposed model. Therefore, an adaptive signal pre-processing approach based on the LUM filter scheme was developed to enhance the fluorescence signal. The reproducibility of DNA quantification for both the machines was also compared by intra-assay as well as inter-assay methods. The comparison can prove whether the present prototype can achieve similar performance as the commercial real-time PCR machine or not.

Without applying the filtering scheme on captured fluorescence images, the measured signal gains of fluorescence intensity at four different initial DNA copies of $X=10^{9}, 10^{7}$, $10^{5}$ and $10^{3}$ order for the prototype are shown in Fig. 5 By the DNA quantification described above, the initial DNA copies of these four samples can be determined as $X m=8 \times 10^{9}$, $2.4 \times 10^{7}, 2 \times 10^{5}$ and $4 \times 10^{3}$. As shown in Fig. 5, a large discrepancy is observed between the predicted and measured fluorescence signal gains for all runs, particularly at the first several cycles in which the fluorescence signal gain is expected to be low. This difference is attributed to the burst noise embedded with the CCD camera. Therefore, an adaptive signal processing scheme is proposed to eliminate the noise for obtaining better results.

Fig. 6 shows the effect of adaptive signal processing on the fluorescence images in six critical cycles to determine DNA quantification. Comparing the images of row data and processed images, it is obvious that the fluorescence signal noise can be eliminated effectively by the adaptive signal processing. The fluorescence signal gain plot obtained by the method is compared with the fluorescence model prediction value in Fig. 7. The model prediction values agree well with the measured data obtained by the real-time PCR prototype after the fluorescence images are processed. The average error with respect to different initial DNA copies is under $6 \%$.

The agreement between the measured data obtained by the adaptive image processing of fluorescence images and the predicted values indicates that the adaptive signal processing 


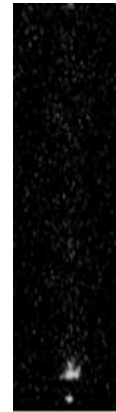

37 cycle

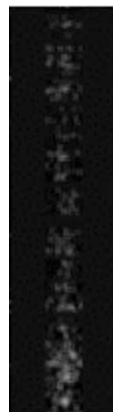

37 cycle

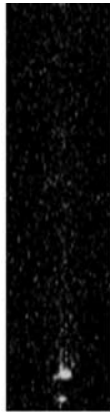

38 cycle

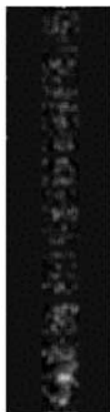

38 cycle

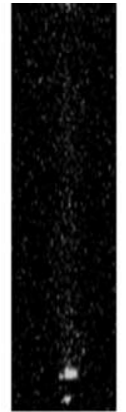

39 cycle



40 cycle

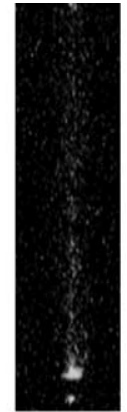

41 cycle

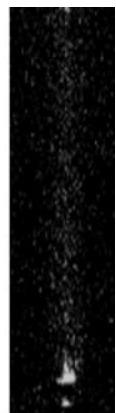

42 cycle

(a)

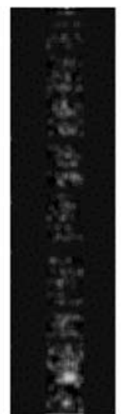

39 cycle

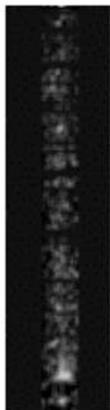

40 cycle

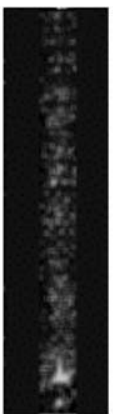

41 cycle

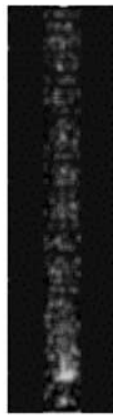

42 cycle

(b)

Fig. 6. Fluorescence images from 37th cycle to 42 nd cycle at initial DNA copies of $10^{5}$ (a) before image filtering process, (b) after image filtering process.

is an effective way to eliminate noise and achieve fidelity for accurate DNA quantification.

The reproducibility of real-time PCR machine depends on factors such as the fluorescence detection system accuracy and the thermal cycling induced PCR efficiency discrepancy. Coefficient of variation (CV) with percentage unit is usually employed as an index and it is defined as:

$\mathrm{CV}=\frac{\log _{10}\left(\sum C_{M} / n\right)-\log _{10}(C s)}{\log _{10}(C s)} 100 \%$

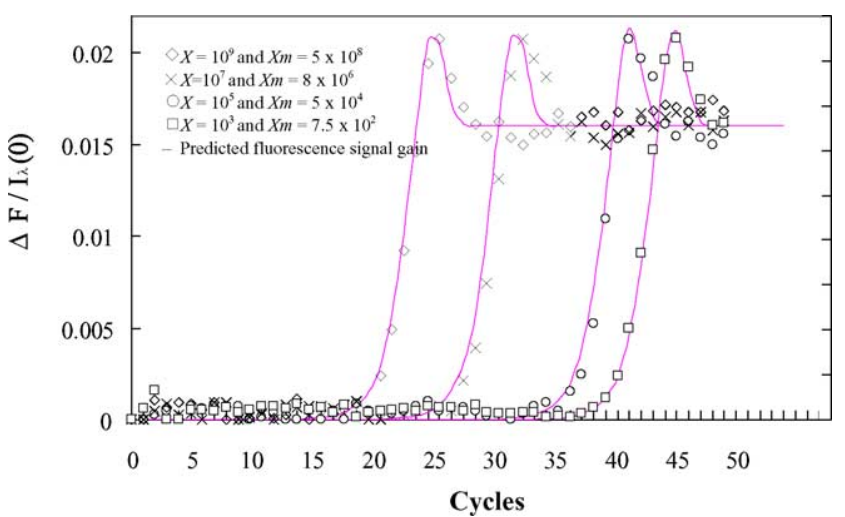

Fig. 7. The comparison of the measured fluorescence intensity from the fluorescence images after processing with three different initial DNA copies denoted in each curve and fluorescence modeling predicted values.
The CV value indicates discrepancy between the average of several quantification results and the value of specified DNA copies. A low CV value means high reproducibility of the system and that all induced measurement errors are minimized.

Fig. 8 shows the $\mathrm{CV}$ value tested by intra-assay with three replicates for different initial DNA copies in the present prototype and the commercial real-time PCR system. Without applying the filtering scheme to the captured images, the quantitative $\mathrm{CV}$ results obtained from the prototype are worse than that from the commercial machine.

However, with filtering scheme, the reproducibility of the prototype can be significantly improved to similar CV values as those obtained from the commercial real-time PCR

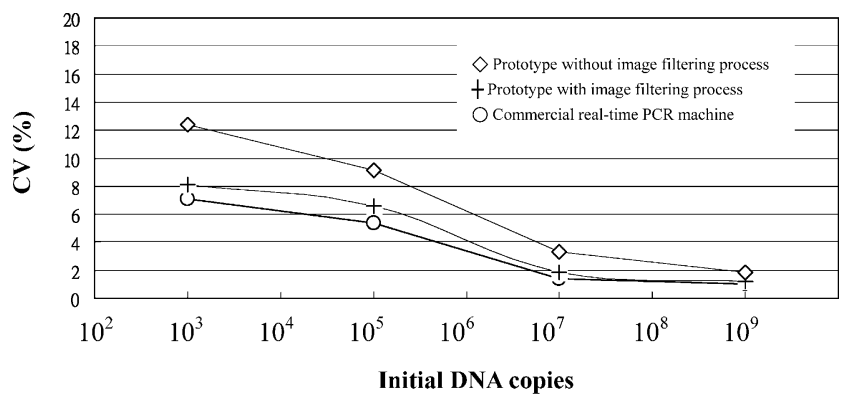

Fig. 8. Measured CV values of real-time PCR prototype and commercial real-time PCR machine for tests with intra-assay using three replicates. 
machine. It proves that a low cost CCD-based fluorescence detection system can achieve good reproducibility in DNA quantification if the captured fluorescence images are filtered with the adaptive image processing scheme developed in this study.

\section{Conclusions}

The following conclusions can be drawn from the measured results and analytical work on two different real-time PCR machines.

1. The real-time PCR prototype with CCD-based fluorimeter was successfully set up. It has a capacity to measure six fluorescence wavelengths at 530, 560, 610, 640, 670 and $710 \mathrm{~nm}$ from the excited fluorescence light from the PCR mix in $20 \mu \mathrm{L}$ volume glass capillary for quantifying initial copies of target DNA in the PCR mix.

2. The analytic models based on Beer-Lambert law are proposed to predict the time history of fluorescence intensity that is measured by the optical sensing system for the proposed prototype.

3. For the prototype, an adaptive image processing algorithm based on LUM filter was developed. After the filtering process on captured images of excited fluorescence from the PCR mix, the CCD detector noise shows a significant reduction.

4. A comparison is made between the real-time PCR prototype and the commercial real-time PCR machine on the accuracy to determine initial copies of HBV SC11 DNA in the PCR mix. Measured results show that both machines have similar accuracy in determination of initial copies of target DNA in the PCR mix when the number of copies is down to $10^{3}$ copies $/ \mathrm{mL}(=0.01 \mathrm{fM})$.

5. The advantages of the prototype over the commercial realtime PCR machine include low-cost and more detecting channels for excited fluorescence intensity of PCR mix. There are only three channels in the commercial realtime PCR but there are six channels in the prototype. For multiplexing real-time PCR, the prototype with six detecting channels can be used to detect initial copies of three different target DNAs (two channels for one target DNA).

6. By software image processing, the simple fluorescence detection system design described in the paper can achieve similar accuracy as the commercial PCR machine with complex optical engine structure. Since computing power is always improving, it gives strong incentive to replace traditional complex optical detection system and highaccuracy optical sensor by simpler structures with appropriate computer signal processing. This will be a good approach to significantly reduce the cost for real-time PCR machine.

\section{Acknowledgement}

The authors deeply appreciate the financial support provided by National Science Council in Taiwan under the grant number of NSC 92-2218-E-002-046.

\section{References}

[1] K.B. Mullis, F. Ferre, R.A. Gibbs, The Polymerase Chain Reaction, Birkhauser, Boston, 1994.

[2] M.A. Innis, D.H. Gelfand, J.J. Shinsky, T.J. White, PCR Protocols: A Guide to Methods and Applications, Academic Press, San Diego, 1990.

[3] R.A. Eeles, A.C. Stamps, Polymerase Chain Reaction (PCR): The Technique and Its Applications, R. G. Landes Co., Austin, TX, 1993.

[4] C.T. Wittwer, M.G. Herrmann, A.A. Moss, R.P. Rasmussen, Continuous fluorescence monitoring of rapid cycle DNA amplification, BioTechniques 22 (1997) 130-138.

[5] C.T. Wittwer, K.M. Ririe, R.V. Andrew, D.A. David, R.A. Gundry, U.J. Balis, The LightCycler ${ }^{\mathrm{TM}}$ : a microvolume multisample fluorimeter with rapid temperature control, BioTechniques 22 (1997) 176-181.

[6] R. Kumaran, R. Kim, A fluorescence based assay for DNA damage by styrene oxide, Sens. Actuat. B 91 (2003) 205-210.

[7] S. Kai, Y. Akira, I. Yutaka, M. Shigeki, M. Hiroaki, A heaterintegrated transparent microchannel chip for continuous flow PCR, Sens. Actuat. B 84 (2002) 283-289.

[8] C.T. Wittwer, M.G. Herrmann, C.N. Gundry, S.J. Kojo, ElenitobeJohnson, Real-time multiplex PCR assays, Methods 25 (2001) 430-442.

[9] http://www.lightcycleronline.com/lc_sys_2/pdfs/flyer.pdf.

[10] A.Q. Liu, X.M. Zhang, V.M. Murukeshan, Q.X. Zhang, Q.B. Zhou, S. Uppili, An optical crossconnect (OXC) using drawbridge micromirrors, Sens. Actuat. A 97-98 (2002) 227-238.

[11] E.S. Kolesar, P.B. Allen, J.W. Wilken, J.T. Howard, Implementation of micromirror arrays as optical binary switches and amplitude modulators, Thin Solid Films 332 (1998) 1-9.

[12] Photonics Industry and Technology Development Association Weekly Report, No. 92078 (2003).

[13] A. Tuantranont, V.M. Bright, J. Zhang, W. Zhang, J.A. Neff, Y.C. Lee, Optical beam steering using MEMS-controllable microlens array, Sens. Actuat. A 91 (2001) 36-372.

[14] http://usa.hamamatsu.com/sys-glossary/highsensitivity.html.

[15] D.-S. Lee, M.-H. Wu, U. Ramesh, C.-W. Lin, T.-M. Lee, H. Chen, A novel real time PCR machine with a miniature spectrometer for fluorescence sensing in a micro liter volume glass capillary, Sens. Actuat. B 100 (2004) 401-410.

[16] C.J. Appelof, E.R. Davidson, Strategies for analyzing data from video fluorometric monitoring of liquid chromatographic effluents, Anal. Chem. 53 (1981) 2053-2056.

[17] D.R. Christmann, S.R. Crouch, A. Timnick, Precision and accuracy of absorption-corrected molecular fluorescence measurements by the cell shift method, Anal. Chem. 53 (1983) 2040-2044.

[18] D.R. Christmann, S.R. Crouch, A. Timnick, Automated instrument for absorption-corrected molecular fluorescence measurements by the cell shift method, Anal. Chem. 53 (1981) 276-280.

[19] J. Knoester, J.E. Van Himbergen, Monte Carlo simulations on concentration self-quenching by statistical traps, J. Chem. Phys. 86 (1987) 3577-3582.

[20] LightCycler Operator's Manual ver. 3.5., 2002.

[21] E. Thomas, K. Gerlinde, P. Birgit, Detection of telomerase components by quantitative real-time on-line PCR analysis with the lightcycler, Biochemica 4 (2000) 16-19. 


\section{Biographies}

Da-sheng Lee was born in Taipei, Taiwan on September 9, 1972. He received $\mathrm{PhD}$ degree from the National Taiwan University in 2004. Afterwards, he joined the department of Air Conditioning and Refrigeration of National Taipei University of Technology as an assistant professor. His main research areas include Lab on a chip for biology, real-time PCR technology, chip type accelerometer and its applications, and MEMS flow sensor.

Bow-How Chang is currently pursuing the $\mathrm{PhD}$ degree in mechanical engineering in National Taiwan University. His current research interests include bio-technology, fluid dynamic bearing and cooling devices for electronic equipments.

Ping-Hei Chen received his Bachelor degree from National Taiwan University in 1980 and $\mathrm{PhD}$ degree from Univ. of Minnesota in 1988. Afterwards, he joined the Dept. of Mechanical Engineering of National Taiwan University as an associate professor. He was promoted to full professor in 1996. He had served as the chairman of the Department of Mechanical Engineering of National Taiwan University from 1998 to 2001. His research areas include micro-thermal-fluid systems, Lab-on-a-chip for DNA detections, Nanofluid, MEMS fabrication technology, and cooling devices for electronic equipments. 